\title{
Fatores associados ao excesso de peso em adultos usuários de restaurantes populares em Belo Horizonte, Brasil
}

\author{
Factors associated with being overweight among adults \\ using low-budget restaurants in Belo Horizonte, Brazil
}

Ísis Eloah Machado ${ }^{1}$

Simone Cardoso Lisboa Pereira ${ }^{1}$

Cláudio Santiago Dias Júnior ${ }^{2}$

Mery Natali Silva Abreu ${ }^{1}$

Angélica Marques Borges ${ }^{1}$

Jullyane Hott Filgueiras ${ }^{1}$

\footnotetext{
${ }^{1}$ Escola de Enfermagem, Universidade Federal de Minas Gerais. Av. Professor Alfredo Balena 190/Escola de Enfermagem, Santa Efigênia. 30.130-100 Belo Horizonte MG Brasil. isiseloah@gmail.com ${ }^{2}$ Faculdade de Filosofia e Ciências Humanas, Universidade Federal de Minas Gerais.
}

\begin{abstract}
The scope of this article is to assess the nutritional status of users of low-budget restaurants and soup kitchens in Belo Horizonte and to identify factors associated with being overweight. It involved a cross-sectional analytical study conducted among adults of both sexes in low-budget restaurants and soup kitchens in Belo Horizonte. Being overweight was the dependent variable and was defined as body mass index (BMI) $\geq$ $25 \mathrm{~kg} / \mathrm{m} 2$. This was correlated with socio-demographic variables and the level of physical activity. The data were obtained directly through weight and height measurement and a semi-structured questionnaire. The study involved 1334 individuals, the majority being male. The prevalence of being overweight was $42.9 \%$ among the men and $45.8 \%$ among the women. The following factors were positively associated with being overweight: being older for both sexes; having a partner for the women; taking less physical exercise and higher socioeconomic level for the men. Detection of factors associated with being overweight reveals that there is a need to implement an effective systematic program of dietary and nutritional education in order to prevent and remedy this condition, so that low-budget restaurants fulfill their objective.
\end{abstract}

Key words Nutritional status, Overweight, Obesity, Socio-demographic factors, Restaurants
Resumo Oobjetivo deste artigo é avaliar o estado nutricional dos usuários dos restaurantes e refeitório populares de Belo Horizonte e identificar fatores associados ao excesso de peso. Estudo analítico transversal, conduzido em adultos de ambos os sexos, nos restaurantes e refeitório populares de Belo Horizonte. O excesso de peso, variável dependente, foi diagnosticado por meio do indice de massa corporal (IMC $\geq 25 \mathrm{~kg} / \mathrm{m}^{2}$ ) e correlacionado com as variáveis sociodemográficas e nivel de atividade física. Os dados foram obtidos por meio da aferição direta do peso e da altura e de questionário semiestruturado. Participaram do estudo 1334 indivíduos, sendo a maioria do sexo masculino. A prevalência de excesso de peso foi de $42,9 \%$ entre os homens e $45,8 \%$ entre as mulheres. Os fatores positivamente associados ao excesso de peso foram: maior idade para ambos os sexos, possuir parceiro para as mulheres e menor prática de atividade física e classe socioeconômica mais elevada para os homens. A identificação dos fatores associados ao excesso de peso revela a necessidade da implantação de uma programação sistemática e eficaz de educação alimentar e nutricional para a prevenção e a remediação desse agravo, para que os restaurantes populares cumpram seu objetivo.

Palavras-chave Estado nutricional, Sobrepeso, Obesidade, Fatores socioeconômicos, Serviços de alimentação 


\section{Introdução}

A alimentação adequada constitui um direito fundamental previsto na Declaração Universal dos Direitos Humanos, proclamada em $1948^{1}$. No Brasil, após uma longa trajetória, o marco histórico na conquista desse direito social na Constituição Federal se deu no ano de 2010, com a Emenda Constitucional $64^{2}$. Embora este direito tenha sido estabelecido, os desvios nutricionais continuam a representar um desafio a ser transposto. Em face desse cenário, o tema da alimentação e nutrição vem sendo objeto de uma intensa reflexão por parte da sociedade civil, dos pesquisadores e do governo brasileiro, ao longo de várias décadas ${ }^{3}$.

De modo a reforçar a perspectiva de criação de um sistema que integre e articule programas, projetos e ações no referido tema, destaca-se o Programa Restaurante Popular, constituído no âmbito do Ministério do Desenvolvimento Social e Combate à Fome ${ }^{4}$. Esse programa tem como objetivo apoiar a implantação e a modernização de restaurantes populares, visando à ampliação de oferta de refeições saudáveis e a preços acessíveis, reduzindo assim, o número de pessoas em situação de insegurança alimentar e vulnerabilidade nutricional. Ademais, o programa preconiza que os restaurantes populares funcionem como espaços multiuso para o desenvolvimento de diversas atividades, tais como a promoção da alimentação saudável da população atendida ${ }^{5,6}$.

Em contraponto, observa-se, atualmente, um aumento da prevalência de sobrepeso e obesidade e a diminuição da ocorrência da desnutrição no Brasil ${ }^{7,8}$. Este fenômeno, denominado transição nutricional, está associado a profundas mudanças econômicas, sociais e demográficas ocorridas nas últimas décadas em decorrência da crescente modernização e urbanização".

Segundo dados da Pesquisa de Orçamentos Familiares (POF) realizada entre 2008 e 2009 pelo Instituto Brasileiro de Geografia e Estatística ${ }^{8}$, a prevalência de excesso de peso e obesidade apresentou elevação em todas as faixas etárias e classes de rendimento da população brasileira. Estima-se uma prevalência de excesso de peso (índice de massa corporal igual ou superior a $25 \mathrm{~kg} /$ $\mathrm{m}^{2}$ ) e obesidade (índice de massa corporal igual ou superior a $30 \mathrm{~kg} / \mathrm{m}^{2}$ ), respectivamente, de $49,0 \%$ e $14,8 \%$ na população adulta brasileira ${ }^{8}$.

Assim, na perspectiva de políticas públicas voltadas a ações de alimentação e nutrição, é relevante destacar que, além do combate à fome, a prevenção de doenças associadas à má alimenta- ção, entre elas a obesidade, também tem sido alvo de preocupação contínua dos órgãos governamentais ${ }^{10}$. Nesse sentido, no ano de 2011, o Ministério da Saúde lançou o Plano Nacional de Enfrentamento das Doenças Crônicas Não Transmissíveis (DCNT), que tem entre seus principais determinantes o excesso de peso e a obesidade, com o objetivo de promover o desenvolvimento e a implementação de políticas públicas efetivas, integradas, sustentáveis e baseadas em evidências para a prevenção e o controle das DCNT e seus fatores de risco, além de fortalecer os serviços de saúde voltados a essas doenças ${ }^{11}$. Outra iniciativa importante, mas que ainda se encontra em fase de planejamento é o Plano Intersetorial de Prevenção e Controle da Obesidade, que se trata de um projeto interministerial, visando a prevenir e controlar a obesidade na população brasileira, por meio de ações intersetoriais, promovendo a alimentação adequada e saudável e a prática de atividades física ${ }^{12}$. Dessa forma, os restaurantes populares devem estar articulados com essas propostas de promover a alimentação saudável e garantir a segurança alimentar e nutricional.

Pioneira na implementação dos restaurantes populares no Brasil, a prefeitura da cidade de Belo Horizonte (MG) inaugurou seu primeiro restaurante em 1994, e, atualmente, conta com quatro unidades funcionais e um refeitório, servindo de modelo para outros restaurantes populares em todo o território nacional ${ }^{13}$. Constituindo um importante cenário para estudo da população que utiliza estes equipamentos.

Diante do exposto, o presente trabalho teve como objetivo avaliar a prevalência de excesso de peso entre os usuários dos restaurantes e refeitório populares de Belo Horizonte e suas possíveis associações com aspectos sociodemográficos e prática de atividade física.

\section{Métodos}

Estudo transversal, de caráter analítico realizado com os usuários dos restaurantes e refeitório populares, do município de Belo Horizonte, Minas Gerais. Este estudo é parte de uma pesquisa intitulada "Prevalência domiciliar de segurança/ insegurança alimentar dos usuários dos restaurantes populares de Belo Horizonte-MG".

Os restaurantes populares de Belo Horizonte, todos analisados neste estudo, fazem parte de uma política pública de inclusão social, que atende uma expressiva parcela da população, ofertando $14 \mathrm{mil}$ refeições/dia. Esses estabelecimentos estão locali- 
zados em áreas centrais da cidade, próximas a locais de transporte de massa e, ou em áreas periféricas com grande aglomeração da população em situação de risco social e nutricional. Atualmente, existem em Belo Horizonte quatro Restaurantes Populares e um Refeitório Popular: Restaurante Popular Herbert de Souza (RPI) - Distrito Sanitário Centro-Sul, Restaurante Popular Josué de Castro (RPII) - Distrito Sanitário Centro-Sul, Restaurante Popular Maria Regina Nabuco (RPIII) - Distrito Sanitário Venda Nova, Restaurante Popular Dom Mauro Bastos (RPIV) - Distrito Sanitário Barreiro, e Refeitório Popular da Câmara Municipal João Bosco Murta Lages (RPV) - Distrito Sanitário Leste.

Os dados foram coletados de setembro de 2009 a fevereiro de 2011. Para isso foi montada uma equipe composta de seis acadêmicas dos Cursos de Graduação em Nutrição e de Gestão de Serviços de Saúde da Universidade Federal de Minas Gerais (UFMG), devidamente treinadas, para aplicar o protocolo de coleta de dados e realizar as aferições antropométricas em todas as unidades, seguindo os critérios da amostragem por $\operatorname{cotas}^{14}$.

Para se estabelecer o tamanho da amostra foi necessário medir o fluxo semanal, uma vez que não existe cadastro dos usuários dos restaurantes populares, apenas o número de refeições vendidas diariamente. A partir desses valores, foi calculada a amostra de usuários de cada estabelecimento ${ }^{14}$ :

$$
\begin{aligned}
& \mathrm{n}_{0}=1 /\left(\mathrm{E}_{0}\right)^{2}(1) \\
& \mathrm{n}=\mathrm{N}^{*} \mathrm{n}_{0} /\left(\mathrm{N}+\mathrm{n}_{0}\right)(2) \\
& \text { Onde: } \\
& \mathrm{n}_{0}=\text { Uma primeira aproxi } \\
& \text { manho da amostra } \\
& \mathrm{N}=\text { Tamanho da populaçã } \\
& \text { usuários por dia) } \\
& \mathrm{E}_{0}=\text { Erro amostral de } 5 \% \\
& \mathrm{n}=\text { Tamanho da amostra }
\end{aligned}
$$

$\mathrm{n}_{0}=$ Uma primeira aproximação para o ta-

$\mathrm{N}=$ Tamanho da população (fluxo médio de

A amostra apurada de adultos usuários dos restaurantes e refeitório populares de Belo Horizonte nos estabelecimentos RPI, RPII RPIII, RPIV e RPV foi de 293, 284, 201, 250 e 284, respectivamente.

Além do cálculo amostral, foi definido que a coleta das informações seria realizada por cotas, respeitando a distribuição por sexo e grupo etário dos usuários e por refeições realizadas em cada estabelecimento. Essa alternativa foi adotada uma vez que não seria possível realizar um sorteio aleatório dos entrevistados ${ }^{14}$. Para fins deste estudo, a amostra da população idosa foi excluída.
Durante a espera para abertura e durante o funcionamento dos estabelecimentos, os usuários foram convidados, depois de questionados se já integravam a pesquisa, a participar de uma entrevista face a face e em seguida de uma avaliação antropométrica.

Os dados sociodemográficos e comportamentais, variáveis independentes, foram obtidos por meio de um questionário semiestruturado previamente testado. Foram incluídos participantes adultos de ambos os sexos. A idade referida em anos completos foi agrupada em cinco faixas etárias: "18-24”; “25-29”; “30-39”; “40-49” e “5059 ” anos. O nível de escolaridade foi classificado segundo o número de anos completos de aprovação na escolaridade formal, em três categorias: "0-8"; "9-11" e "12 ou mais". Os participantes foram também classificados segundo estado conjugal, sendo duas categorias: "sem parceiro" e "com parceiro". A cor da pele foi autorreferida por meio das opções branca, preta, parda, amarela ou indígena e posteriormente foi categorizada em "branca" ou "não branca", possuir filhos foi categorizado em: “sim” e "não." Para classificação econômica, adotou-se o Critério de Classificação Econômica Brasil (CCEB), que estima o poder de compra das pessoas e famílias urbanas, abandonando a pretensão de classificar a população em termos de "classes sociais"15.

A atividade física no lazer foi caracterizada em: "todos os dias" para a prática de pelo menos 30 minutos de atividade física de intensidade leve ou moderada (caminhada, caminhada em esteira, musculação, hidroginástica, ginástica em geral, natação, artes marciais e luta, bicicleta, voleibol ou outra) por pelo menos 30 minutos diários em 5 ou mais dias da semana; " 2 a 4 vezes por semana" para atividades físicas realizadas de 2 a 4 vezes por semana; e "Não" para aqueles que fazem atividade física uma vez por semana ou menos.

Os dados antropométricos (peso e altura) foram coletados por entrevistadores devidamente treinados. Para a medida do peso foi utilizada uma balança digital da marca Plenna ${ }^{\circledR}$, com capacidade para $150 \mathrm{Kg}$ e precisão de 100 g. A estatura foi aferida utilizando um estadiômetro da marca Alturexata ${ }^{\circledR}$, com precisão de $1 \mathrm{~mm}$.

O estado nutricional, variável dependente deste estudo, foi definido com base na avaliação antropométrica do índice de massa corporal (IMC), expresso pela fórmula IMC $=[$ peso $/($ al tura $)^{2}$. O IMC foi tratado sob a forma contínua, sendo posteriormente agrupado em quatro categorias, segundo as recomendações da Organização Mundial da Saúde (OMS) ${ }^{16}$ : "baixo peso" 
(IMC menor que $18,5 \mathrm{Kg} / \mathrm{m}^{2}$ ), "eutrofia" (IMC entre 18,5 e 24,9 Kg/m²), "sobrepeso" (IMC entre 25 e $29,9 \mathrm{Kg} / \mathrm{m}^{2}$ ) e obesidade (IMC maior ou igual a $\left.30 \mathrm{Kg} / \mathrm{m}^{2}\right)^{16}$. Posteriormente, "baixo peso" e "eutrofia" foram agrupados e comparados com a categoria "excesso de peso", utilizada como desfecho de interesse para as análises, que foi resultado do agrupamento das categorias "sobrepeso" e "obesidade".

O banco de dados foi criado utilizando o software EpiData versão 3.1.2, com dupla digitação e avaliação da consistência dos dados.

Com o auxílio do software Statistical Package for the Social Sciences (SPSS) v.19.0 for Windows foi realizada a análise descritiva, com cálculo das frequências para variáveis categóricas e cálculo de média e desvio padrão para variáveis contínuas com distribuição normal. O teste de Kolmogorov-Smirnov foi utilizado para avaliação da aderência das variáveis à distribuição normal. $\mathrm{Na}$ análise univariada, foram utilizados o $\mathrm{t}$ de Student simples para comparação de médias das amostras independentes e qui-quadrado de Pearson para avaliar a diferença entre proporções. Adotou-se nível de significância de 5\% (p < 0,05).

As variáveis $\operatorname{com} p<0,20$ na análise univariada foram selecionadas para a análise multivariada por meio da regressão de Poisson com variâncias robustas. Calcularam-se as razões de prevalência ajustadas e os respectivos intervalos de confiança de $95 \%$. Os modelos foram estratificados por gênero. Permaneceram no modelo final as variáveis significativas ao nível de significância de 5\%. Para a análise multivariada foi utilizado o aplicativo STATA versão 9.2 (Statistical Analysis System Software).

Os participantes do estudo receberam informações detalhadas sobre a avaliação e assinaram um termo de consentimento livre e esclarecido. O projeto, no qual este estudo está inserido, foi aprovado pelo Comitê de Ética em Pesquisa da Universidade Federal de Minas Gerais (UFMG).

\section{Resultados}

Participaram do estudo 1334 indivíduos adultos, sendo 954 (71,5\%) do sexo masculino, com média de idade de $36,4 \pm 11,3$ anos. O número de entrevistas realizadas e aferições antropométricas atenderam à amostra calculada para todas as unidades avaliadas, sendo que no RPI, RPII RPIII, RPIV e RPV o número atingido foi de 305 , 299, 186, 264 e 280, respectivamente.
Um total de 1334 indivíduos passou pela avaliação antropométrica do estado nutricional, os quais foram considerados na análise desse artigo. A prevalência de sobrepeso foi de $32,2 \%$ e de obesidade $11,3 \%$. Observou-se diferença significativa na distribuição do estado nutricional entre os sexos (valor-p $<0,001$ ). A prevalência de sobrepeso foi mais elevada entre os homens $(33,4 \%)$ do que entre as mulheres $(29,2 \%)$, ocorrendo o inverso em relação à obesidade, $16,6 \%$ para as mulheres e $9,5 \%$ para os homens. Ao avaliar o IMC de forma numérica, observou-se que a média total foi de $24,9 \pm 4,4 \mathrm{Kg} / \mathrm{m}^{2}$, sendo que a média para as mulheres $\left(25,4 \pm 5,3 \mathrm{Kg} / \mathrm{m}^{2}\right)$ foi maior do que para os homens $(24,8 \pm 4,0 \mathrm{Kg} /$ $\left.\mathrm{m}^{2}\right)(\mathrm{p}=0,01)($ Tabela 1$)$.

Quando se analisa os dados sociodemográficos, no que se refere à idade, observa-se que não houve diferença significativa entre os sexos. A média de idade foi de 36 anos $( \pm 11)$ entre os homens e de 37 anos $( \pm 12)$ entre as mulheres. Foi encontrada maior frequência de mulheres com filhos $(62,5 \%)$ em comparação aos homens $(52,5 \%)(\mathrm{p}<0,001)$ (Tabela 1).

No que se refere à classe econômica, mais de $50 \%$ dos homens e das mulheres eram provenientes da classe $\mathrm{C}$, no entanto, para os homens a segunda classe econômica de maior prevalência foi a A e B, já para as mulheres foi a D e E (Tabela 1).

Com relação ao nível de atividade física diária, $42,6 \%$ dos homens relataram praticar pelo menos 30 minutos de atividade física no tempo livre todos os dias da semana, já entre as mulheres, somente $27,2 \%$ relataram essa prática, diferença estatisticamente significativa $(\mathrm{p}<0,001)$. A prática de atividade física pelo menos 30 minutos de duas a quatro vezes por semana foi relatada por $13,5 \%$ dos homens e $9,5 \%$ das mulheres (Tabela 1).

Os resultados da análise univariada indicam que idade mais avançada, menor escolaridade, possuir parceiro e ter filhos são fatores que estiveram associados $(p<0,05)$ ao excesso de peso tanto para os homens como para as mulheres. Além dessas variáveis, para os homens, a classe econômica mais alta (A ou B) também mostrou associação com o desfecho analisado (Tabela 2).

$\mathrm{Na}$ análise multivariada, as variáveis que permaneceram no modelo final, associadas ao excesso de peso foram: idade para ambos os sexos, estado conjugal para o sexo feminino e classe econômica e nível de atividade física para os homens (Tabela 3).

Mesmo após o ajuste, uma maior idade manteve-se associada a uma maior probabilidade de 
Tabela 1. Distribuição da população adulta usuária dos restaurantes e refeitório populares de Belo Horizonte (MG), segundo estabelecimentos estudados, estado nutricional, características sociodemográficas e prática de atividade física por sexo. 2009-2011. $(\mathrm{n}=1334)$ 2009-2011. $(\mathrm{n}=1334)$.

\begin{tabular}{|c|c|c|c|c|}
\hline \multirow[b]{2}{*}{ Variáveis } & \multicolumn{2}{|c|}{ Homens } & \multicolumn{2}{|c|}{ Mulheres } \\
\hline & $\mathbf{n}$ & $\%$ & $\mathbf{n}$ & $\%$ \\
\hline \multicolumn{5}{|l|}{ Estado nutricional $(\mathrm{n}=1.334)^{*}$} \\
\hline Baixo peso & 24 & 2,5 & 24 & 6,3 \\
\hline Eutrófico & 520 & 54,5 & 182 & 47,9 \\
\hline Sobrepeso & 319 & 33,4 & 111 & 29,2 \\
\hline Obesidade & 91 & 9,5 & 63 & 16,6 \\
\hline \multicolumn{5}{|l|}{ Estabelecimento $(\mathrm{n}=1.334)^{*}$} \\
\hline RPI & 240 & 25,2 & 65 & 17,1 \\
\hline RPII & 191 & 20,0 & 108 & 28,4 \\
\hline RPIII & 146 & 15,3 & 40 & 10,5 \\
\hline RPIV & 196 & 20,5 & 68 & 17,9 \\
\hline RPV & 181 & 19,0 & 99 & 26,1 \\
\hline \multicolumn{5}{|l|}{ Idade $(\mathrm{n}=1.334)$} \\
\hline 18 a 24 anos & 155 & 16,2 & 67 & 17,6 \\
\hline 25 a 29 anos & 175 & 18,3 & 57 & 15,0 \\
\hline 30 a 39 anos & 268 & 28,1 & 90 & 23,7 \\
\hline 40 a 49 anos & 219 & 23,0 & 94 & 24,7 \\
\hline 50 a 59 anos & 137 & 14,4 & 72 & 18,9 \\
\hline \multicolumn{5}{|l|}{ Estado conjugal $(\mathrm{n}=1.329)$} \\
\hline Sem parceiro & 574 & 60,4 & 221 & 58,5 \\
\hline Com parceiro & 377 & 39,6 & 157 & 41,5 \\
\hline \multicolumn{5}{|l|}{ Possui filhos $(\mathrm{n}=1.333)^{*}$} \\
\hline Sim & 501 & 52,5 & 237 & 62,5 \\
\hline Não & 453 & 47,5 & 142 & 37,5 \\
\hline \multicolumn{5}{|l|}{ Escolaridade $(\mathrm{n}=1.330)^{*}$} \\
\hline 0 a 8 anos & 183 & 19,2 & 145 & 38,3 \\
\hline 9 a 11 anos & 559 & 58,8 & 178 & 47,0 \\
\hline 12 anos ou mais & 209 & 22,0 & 56 & 14,8 \\
\hline \multicolumn{5}{|l|}{ Classe econômica $(\mathrm{n}=1.078)^{*}$} \\
\hline Classes A e B & 222 & 28,7 & 68 & 22,4 \\
\hline Classe C & 423 & 54,7 & 165 & 54,3 \\
\hline Classes D e E & 129 & 16,7 & 71 & 23,4 \\
\hline \multicolumn{5}{|l|}{ Cor da pele $(\mathrm{n}=1.262)$} \\
\hline Branca & 292 & 32,1 & 119 & 33,7 \\
\hline Não branca & 617 & 67,9 & 234 & 66,3 \\
\hline \multicolumn{5}{|c|}{ Nível de atividade física diária* $(\mathrm{n}=1.332)$} \\
\hline Todos os dias & 406 & 42,6 & 103 & 27,2 \\
\hline De 2 a $4 x /$ semana & 129 & 13,5 & 36 & 9,5 \\
\hline Não & 418 & 43,9 & 240 & 63,3 \\
\hline Total & 953 & 71,55 & 379 & 28,45 \\
\hline
\end{tabular}

*Houve diferença significativa para as variáveis entre os sexos $(\mathrm{p}<0,05)$.

excesso de peso. Observou-se um gradiente doseresposta, isto é, com o aumento da idade houve aumento gradativo da RP para ambos os sexo. Para o sexo feminino, observou-se que possuir parceiro aumenta em $38 \%$ a probabilidade de possuir excesso de peso em relação às que não possuem ( $\mathrm{RP}=1,38$; IC 95\%: 1,11-1,71). Para a população masculina, não praticar atividade fí- sica aumentou em 24\% a probabilidade de ter excesso de peso ( $\mathrm{RP}=1,24$; IC 95\% 1,04-1,47) em relação aos que praticam atividades físicas diariamente. Constatou-se, também, que há uma elevação na prevalência de excesso de peso quanto maior for a classe econômica, sendo que, em comparação com as classes D e E, as classes A e B apresentam probabilidade 54\% maior de apre- 
Tabela 2. Prevalência de excesso de peso (IMC maior que $25 \mathrm{Kg} / \mathrm{m}^{2}$ ) segundo variáveis socioeconômicas e nível de atividade física, de acordo com o sexo, da população adulta usuária dos restaurantes e refeitório populares de Belo Horizonte, MG. 2009-2011. ( $\mathrm{n}=1134$ ).

\begin{tabular}{|c|c|c|c|c|c|c|c|c|c|c|}
\hline \multirow{3}{*}{ Variáveis } & \multicolumn{5}{|c|}{ Homens } & \multicolumn{5}{|c|}{ Mulheres } \\
\hline & \multicolumn{2}{|c|}{ Não } & \multicolumn{2}{|c|}{ Sim } & \multirow[b]{2}{*}{$\mathbf{p}$} & \multicolumn{2}{|c|}{ Não } & \multicolumn{2}{|c|}{ Sim } & \multirow[b]{2}{*}{ p } \\
\hline & $\mathbf{n}$ & $\%$ & $\mathbf{n}$ & $\%$ & & $\mathbf{n}$ & $\%$ & $\mathbf{n}$ & $\%$ & \\
\hline Estabelecimento & & & & & 0,39 & & & & & 0,38 \\
\hline RPI & 141 & 58,8 & 99 & 41,3 & & 37 & 56,9 & 28 & 43,1 & \\
\hline RPII & 114 & 59,7 & 77 & 40,3 & & 56 & 51,9 & 52 & 48,1 & \\
\hline RPIII & 87 & 59,6 & 59 & 40,4 & & 19 & 47,5 & 21 & 52,5 & \\
\hline RPIV & 100 & 51,0 & 96 & 49,0 & & 33 & 48,5 & 35 & 51,5 & \\
\hline RPV & 102 & 56,4 & 79 & 43,6 & & 61 & 61,6 & 38 & 38,4 & \\
\hline Idade & & & & & $<0,01$ & & & & & $<\mathbf{0 , 0 1}$ \\
\hline 18 a 24 anos & 120 & 77,4 & 35 & 22,6 & & 50 & 74,6 & 17 & 25,4 & \\
\hline 25 a 29 anos & 112 & 64,0 & 63 & 36,0 & & 36 & 63,2 & 21 & 36,8 & \\
\hline 30 a 39 anos & 146 & 54,5 & 122 & 45,5 & & 50 & 555,6 & 40 & 44,4 & \\
\hline 40 a 49 anos & 103 & 47,0 & 116 & 53,0 & & 46 & 48,9 & 48 & 51,1 & \\
\hline 50 a 59 anos & 63 & 46,0 & 74 & 54,0 & & 24 & 33,3 & 48 & 66,7 & \\
\hline Escolaridade & & & & & $<0,01$ & & & & & 0,01 \\
\hline 0 a 8 anos & 67 & 36,6 & 116 & 63,4 & & 65 & 44,8 & 80 & 55,2 & \\
\hline 9 a 11 anos & 334 & 59,7 & 225 & 40,3 & & 108 & 60,7 & 70 & 39,3 & \\
\hline 12 anos ou mais & 141 & 67,5 & 68 & 43,0 & & 33 & 58,9 & 23 & 41,1 & \\
\hline Estado conjugal & & & & & $<0,01$ & & & & & $<0,01$ \\
\hline Sem parceiro & 367 & 63,9 & 207 & 36,1 & & 136 & 61,5 & 85 & 38,5 & \\
\hline Com parceiro & 175 & 46,4 & 202 & 53,6 & & 68 & 43,3 & 89 & 56,7 & \\
\hline Possuir filhos & & & & & $<0,01$ & & & & & $<0,01$ \\
\hline Sim & 261 & 52,1 & 240 & 47,9 & & 107 & 45,1 & 130 & 54,9 & \\
\hline Não & 283 & 62,5 & 170 & 37,5 & & 98 & 69,0 & 44 & 31,0 & \\
\hline Classe econômica & & & & & 0,01 & & & & & 0,68 \\
\hline Classes A e B & 111 & 50,0 & 111 & 50,0 & & 40 & 58,8 & 28 & 41,2 & \\
\hline Classe C & 241 & 57,0 & 182 & 43,0 & & 88 & 53,3 & 77 & 46,7 & \\
\hline Classes D e E & 86 & 66,7 & 43 & 33,3 & & 37 & 52,1 & 34 & 47,9 & \\
\hline Cor da pele & & & & & 0,58 & & & & & 0,77 \\
\hline Branca & 169 & 57,9 & 123 & 42,1 & & 65 & 54,6 & 54 & 45,4 & \\
\hline Não-branca & 345 & 55,9 & 272 & 44,1 & & 124 & 53,0 & 110 & 47,0 & \\
\hline Nível de atividade física diária & & & & & 0,08 & & & & & 0,86 \\
\hline Todos os dias & 248 & 61,1 & 158 & 38,9 & & 57 & 55,3 & 46 & 44,7 & \\
\hline De 2 a $4 x /$ semana & 72 & 55,8 & 57 & 44,2 & & 18 & 50,0 & 18 & 50,0 & \\
\hline Não & 223 & 53,3 & 195 & 46,7 & & 130 & 54,2 & 110 & 45,8 & \\
\hline
\end{tabular}

sentar o agravo $(\mathrm{RP}=1,54 ; \mathrm{IC} 95 \% 1,16-2,03)$ (Tabela 3).

\section{Discussão}

A caracterização dos usuários dos restaurantes e refeitório populares, a partir da avaliação antropométrica do estado nutricional e a identificação de fatores associados aos desvios nutricionais são relevantes, uma vez que a alimentação oferecida por estes locais deve ser capaz de garantir aporte nutricional adequado à população, bem como incorporar ações de promoção da alimentação saudável ${ }^{10,17}$. Neste sentido, as informações acerca da prevalência de excesso de peso e fatores associados a esse agravo, em meio à população atendida, podem apontar para a necessidade premente de realizar ações de promoção de práticas alimentares saudáveis nesses espaços, que respeitem as diferenças e que sejam social, econômica e ambientalmente sustentáveis, em consonância com a proposta do Programa.

A OMS ${ }^{16}$ considera o sobrepeso e a obesidade como os principais problemas de saúde pública da população mundial, atingindo todas as 
Tabela 3. Análise multivariada para fatores associados ao excesso de peso no sexo feminino e masculino, com suas respectivas razões de prevalência e intervalo de confiança de 95\%, Belo Horizonte (MG). 2009-2011.

\begin{tabular}{|c|c|c|c|c|}
\hline \multirow{3}{*}{ Variável } & \multicolumn{4}{|c|}{ Excesso de peso } \\
\hline & \multicolumn{2}{|c|}{ Homens } & \multicolumn{2}{|c|}{ Mulheres } \\
\hline & $\mathbf{R P}$ & IC95\% & $\mathbf{R P}$ & IC95\% \\
\hline \multicolumn{5}{|l|}{ Idade (anos) } \\
\hline 18 a 24 anos & 1,00 & - & 1,00 & - \\
\hline 25 a 29 anos & 1,65 & {$[1,10 ; 2,45]$} & 1,40 & {$[0,83 ; 2,37]$} \\
\hline 30 a 39 anos & 2,03 & {$[1,41 ; 2,92]$} & 1,60 & {$[0,99 ; 2,59]$} \\
\hline 40 a 49 anos & 2,39 & {$[1,66 ; 3,44]$} & 1,81 & {$[1,14 ; 2,87]$} \\
\hline 50 a 59 anos & 2,49 & {$[1,70 ; 3,63]$} & 2,46 & {$[1,58 ; 3,83]$} \\
\hline \multicolumn{5}{|l|}{ Estado conjugal } \\
\hline Sem parceiro & - & - & 1,00 & - \\
\hline Com parceiro & - & - & 1,38 & {$[1,11 ; 1,71]$} \\
\hline \multicolumn{5}{|l|}{ Classe econômica } \\
\hline Classes A e B & 1,54 & {$[1,17 ; 2,03]$} & - & - \\
\hline Classe C & 1,35 & {$[1,03 ; 1,76]$} & - & - \\
\hline Classes D e E & 1,00 & - & - & - \\
\hline \multicolumn{5}{|c|}{ Nível de atividade física diária } \\
\hline Todos os dias & 1,00 & - & - & - \\
\hline De 2 a $4 x /$ semana & 1,07 & {$[0,84 ; 1,36]$} & - & - \\
\hline Não & 1,24 & {$[1,04 ; 1,47]$} & - & 一 \\
\hline
\end{tabular}

faixas etárias. No presente estudo, ao analisar o estado nutricional, verificou-se que as prevalências de excesso de peso na população adulta estudada evidenciam os níveis epidêmicos deste problema. Enquanto ocorre baixa prevalência de desnutrição, uma elevada parcela da população apresentou sobrepeso e obesidade, sendo que a prevalência de excesso de peso ultrapassou em quase 12 vezes o baixo peso. Resultado similar foi encontrado por Barbosa et al. ${ }^{18}$ em um estudo transversal realizado em população de baixa renda no Nordeste brasileiro. Verificaram que $5,9 \%$ da população adulta estudada apresentou baixo peso, ao passo que $41,2 \%$ mostraram sobrepeso e obesidade ${ }^{18}$. Estas tendências também foram encontradas por outros estudos nacionais, como o da Pesquisa de Orçamentos Familiares (POF) realizada entre 2008 e 2009 pelo Instituto Brasileiro de Geografia e Estatística, em que 2,7\% da população adulta avaliada apresentava déficit de peso enquanto o excesso de peso atingiu cerca da metade da população dos homens e das mulheres, 50,1 e 48,0\% respectivamente ${ }^{8}$.

Ao analisar os desvios nutricionais estratificados por sexo, verifica-se que as prevalências de déficit de peso e obesidade são significativamente maiores entre as mulheres. A POF 2008-20098 mostra que a obesidade atinge $16,0 \%$ e o déficit de peso 3,6\% das brasileiras, resultados semelhantes aos encontrados pela presente pesquisa. Por outro lado, a prevalência de obesidade entre homens ficou abaixo da média nacional $(12,5 \%)$ e a de déficit de peso foi semelhante (2-3\%). Destaca-se que a prevalência de déficit de peso em mulheres usuárias dos restaurantes e refeitório populares de Belo Horizonte (MG) foi semelhante apenas à encontrada entre mulheres de domicílios rurais da Região Nordeste do Brasil ${ }^{8}$. Ressalta-se que a população estudada na POF não é completamente comparável à do presente estudo devido, principalmente, a diferenças na distribuição de classes socioeconômicas e de faixas etárias. Assim, a população usuária do restaurante exige atenção especial das políticas sociais, pois se apresentam mais vulneráveis quanto à sua saúde e não somente às carências nutricionais e doenças infecto-parasitárias, mas também à obesidade e às doenças crônicas.

Em face da prevalência de excesso de peso observada, verifica-se que a população estudada segue a tendência brasileira, enquadrando-se como grupo alvo de ações de saúde destinadas ao controle de doenças e agravos não transmissíveis. Ademais, enfatiza-se, em conformidade com a Estratégia Global da Organização Mundial da Saúde (OMS) para Alimentação, Ativida- 
de Física e Saúde, a necessidade de adequação dos padrões de alimentação desses usuários, um dos determinantes desse agravo ${ }^{19}$. Essa demanda é ainda mais salientada, visto os dados de um estudo recente ${ }^{20}$, que apontam as características negativas da qualidade da dieta da população brasileira observadas ao final da primeira década do século XXI, em todas as regiões, a saber: excesso de açúcares livres e gorduras; proporção elevada de gorduras saturadas, com maior participação de produtos de origem animal; a presença insuficiente de frutas, verduras e legumes; e a intensificação do teor de gorduras e diminuição do de carboidratos ${ }^{20}$.

Em relação ao efeito da idade sobre a prevalência de excesso de peso, os resultados obtidos corroboram a literatura. Barbosa et al. ${ }^{18}$ evidenciaram que a prevalência de excesso de peso de homens e mulheres de maior idade ( $>40$ anos) apresentou-se maior do que a dos mais jovens. Segundo dados da $\mathrm{POF}^{8}$, as maiores prevalências foram encontradas acima dos 35 anos em ambos os sexos. Em estudo realizado por Sá e Moura $^{21}$, a prevalência de excesso de peso na população masculina chegou a atingir $73,7 \%$ da população com idade $\geq 55$ anos e na população feminina $58,3 \%$ da população com idade $\geq 55$ anos. A relação positiva entre a idade e o excesso de peso poderia ser elucidada, em parte, pela redução da taxa metabólica basal que fisiologicamente ocorre no processo de envelhecimento ${ }^{22} \mathrm{e}$ pela redução da prática de exercícios físicos ${ }^{23}$.

No que diz respeito ao estado civil, observou-se que o fato de ter parceiro influenciou na prevalência de excesso de peso em mulheres usuárias dos estabelecimentos deste estudo. Resultado similar foi observado por outros autores ${ }^{24-27}$ e são sugestivos de que ocorrem mudanças no estilo de vida em decorrência da alteração no estado conjugal, de solteiro(a) para casado(a), entre as quais se destaca a piora dos hábitos alimentares, redução na prática de atividade física e maior propensão a deixar o tabagismo ${ }^{28}$.

A escolaridade não se manteve associada ao excesso de peso no modelo final para nenhum dos sexos e esta relação ainda é controversa na literatura. Alguns estudos encontraram associação inversa entre escolaridade e excesso de peso entre mulheres ${ }^{24-26,29-31}$, enquanto, entre homens, encontraram relação direta entre escolaridade e excesso de peso $^{27,29,31}$ e outros ainda não encontraram nenhuma associação como no presente estudo ${ }^{25,26,31,32}$.

Embora os restaurantes populares se destinem ao público de baixa renda e estudo recente tenha demonstrado que $27,7 \%$ da população aten- dida pelos restaurantes populares de Belo Horizonte esteja em situação de insegurança alimentar e nutricional ${ }^{33}$, foram observadas distintas classes econômicas na população estudada, fator que influenciou o excesso de peso entre os homens. Aqueles de melhor classe econômica apresentaram maior percentual de excesso de peso. Não foram encontrados outros estudos que utilizaram a mesma metodologia para classificação econômica, entretanto há autores que correlacionaram estado nutricional à renda, sendo que os estudos realizados no Brasil têm encontrado maior prevalência de sobrepeso e obesidade para homens com maior renda domiciliar e o inverso para as mulheres ${ }^{25,34}$.

Algumas das razões apontadas para a relação direta entre nível econômico e excesso de peso nos homens se referem à proteção natural contra a enfermidade que seria encontrada entre os estratos sociais menos favorecidos em face da escassa disponibilidade de alimentos e do perfil de intensa atividade física que seriam característicos dos mesmos ${ }^{25}$.

No que tange à prática de atividade física, os resultados encontrados não são muito bem esclarecidos quando comparados entre os sexos. Em estudo transversal com base em dados secundários obtidos pelo VIGITEL, no ano de $2008^{27}$, e em estudo com moradores do município de Duque de Caxias ${ }^{35}$, também foi encontrada associação entre inatividade física e excesso de peso somente no sexo masculino, tal como observado no presente estudo. Por outro lado, em diversos estudos observou-se relação positiva entre excesso de peso e inatividade física apenas em mulheres ${ }^{25,26,31}$. Esses achados demonstram que as diferenças nas práticas de atividade física encontradas podem indicar que programas específicos considerando o gênero devam ser planejados para a redução e prevenção do ganho de peso'.

Um aspecto importante deste estudo relaciona-se ao fato de que o peso e a altura puderam ser aferidos, tendo em vista que em estudos nacionais como, por exemplo, o VIGITEL esses parâmetros são autorreferidos ${ }^{7}$. O peso corporal referido é frequentemente subestimado e a altura, superestimada em relação às medidas aferidas, influenciando negativamente o IMC ${ }^{36}$.

Destaca-se que embora tenham ocorrido perdas, principalmente devido à recusa em participar das aferições antropométricas, a amostra utilizada ficou muito próxima da calculada, superando $90 \%$ do esperado em todas as unidades, não ocasionando prejuízo à representatividade do estudo. 
O estudo do tipo transversal utilizado tem como limitação o fato das medições serem feitas no mesmo período, espaço e tempo, não podendo distinguir causa-efeito do problema. Ressalta-se, entretanto, que o objetivo deste trabalho foi verificar a prevalência de excesso de peso e identificar possíveis associações com as características sociodemográficas e a prática de atividade física. Assim, são necessários estudos de caráter longitudinal para verificação dos fatores associados ao aumento da prevalência de excesso de peso nessa população.

Ressalta-se que a metodologia empregada no presente estudo não permite detectar carências nutricionais e nem o verdadeiro grau de segurança alimentar dos usuários, sendo necessários mais estudos para melhor descrever o estado nutricional da população. Entretanto, os resultados apontam a importância de ações de educação nutricional e de pesquisa que examinem a composição do cardápio e das porções oferecidos, em relação a padrões estabelecidos pelo Programa Restaurantes Populares e recomendações para uma alimentação saudável, a fim de reduzir a prevalência de excesso de peso na população.

Em suma, a prevalência de excesso de peso encontrada entre os usuários dos restaurantes e refeitório populares, a elevada prevalência de sobrepeso entre os homens e de obesidade entre as mulheres já podem ser contempladas como um problema de Saúde Pública nesta população, indicando que é necessário adotar medidas que possam reverter o crescimento da prevalência deste agravo.

\section{Conclusão}

O presente estudo demonstrou que os usuários dos restaurantes e refeitório populares de Belo Horizonte apresentaram estado nutricional similar ao encontrado no Brasil, em que a elevada prevalência de excesso de peso entre ambos os sexos, aponta um problema de saúde pública nestes locais.

$\mathrm{O}$ aumento da idade, a reduzida prática de atividade física e pertencer à classe econômica mais elevada foram fatores que influenciaram positivamente o excesso de peso nos homens. Já para as mulheres o excesso de peso foi influenciado pela idade mais avançada e possuir parceiro. Ademais, atenção especial deve ser direcionada à prevalência de déficit de peso entre as mulheres, visto ter sido elevada.

Dessa forma, este estudo contribuiu para melhor entendimento sobre os fatores que se associam ao excesso de peso na população, o que poderá subsidiar o planejamento de ações direcionadas à prevenção e ao manejo deste agravo, nos espaços dos restaurantes populares, como uma das estratégias para a promoção da segurança alimentar e nutricional.

Os Restaurantes Populares, bem como outros programas de segurança alimentar devem promover a alimentação saudável conforme suas diretrizes e o que as recentes propostas governamentais de promoção da saúde preconizam, sendo necessários estudos de monitoramento e avaliação, observando-se os impactos, a eficiência das ações e a cobertura dos mesmos.

\section{Colaboradores}

IE Machado, JH Filgueiras e AM Borges participaram na coleta de dados, análise estatística, discussão dos dados e redação do artigo. MNS Abreu participou na análise e discussão dos dados e redação do artigo. CS Dias Júnior participou na elaboração do projeto, no cálculo amostral de usuários de cada estabelecimento e na revisão do artigo. SCL Pereira participou na elaboração do projeto, coordenação da coleta de dados e revisão do artigo. 


\section{Referências}

1. Organização das Nações Unidas (ONU). Declaração Universal dos Direitos Humanos. Nova York: ONU; 1948. [Internet]. [acessado 2011 maio 10]. Disponível em: http://portal.mj.gov.br/sedh/ct/ legis_intern/ddh_bib_inter_universal.htm

2. Custódio MB, Yuba TY, Cyrillo DC. Política de segurança alimentar e nutricional no Brasil: uma análise da alocação de recursos. Rev Panam Salud Publica 2013; 33(2):144-150.

3. Recine E, Vasconcellos AB. Políticas nacionais e o campo da Alimentação e Nutrição em Saúde Coletiva: cenário atual. Cien Saude Colet 2011; 16(1):73-79.

4. Brasil. Ministério do Desenvolvimento Social e Combate à Fome (MDS). Relatório Final da III Conferência Nacional de Segurança Alimentar e Nutricional. Brasília: MDS; 2007.

5. Rose DD. Interventions to reduce household food insercurity: a synthesis of current concepts and approaches for Latin America. Rev Nutr 2008; 21(Supl.):159-173.

6. Brasil. Ministério do Desenvolvimento Social e Combate a Fome (MDS). Manual Programa Restaurante Popular. Brasília: MDS; 2004.

7. Brasil. Ministério da Saúde (MS). Vigitel Brasil 2010 - Vigilância de Fatores de Risco e Proteção para Doenças Crônicas por Inquérito Telefônico. Brasília: MS; 2011.

8. Instituto Brasileiro de Geografia e Estatística (IBGE). Ministério da Saúde e Ministério do Planejamento. Orçamento e Gestão. Pesquisa de Orçamentos Familiares 2008-2009: Antropometria e Estado Nutricional de Crianças, Adolescentes e Adultos no Brasil. Rio de Janeiro: IBGE; 2010.

9. Sichieri R, Moura EC. Análise multinível das variações no índice de massa corporal entre adultos, Brasil, 2006. Rev Saude Publica 2009; 43(Supl. 2):90-97.

10. Souza LM. Fatores associados à insegurança alimentar nos domicílios da Região Nordeste do Brasil, 2004 [tese]. Belo Horizonte: Universidade Federal de Minas Gerais; 2009.

11. Malta DC, Dimech CPN, Moura L, Silva Júnior JB. Balanço do primeiro ano da implantação do Plano de Ações Estratégicas para o Enfrentamento das Doenças Crônicas Não Transmissíveis no Brasil, 2011 a 2022. Epidemiol. Serv. Saúde. 2013; 22(1):171-178.

12. Brasil. Ministério do Desenvolvimento Social e Combate à Fome (MDS). Conselho Nacional de Segurança Alimentar e Nutricional (CONSEA). Exposição de motivos no 008-2012. Brasília: Consea; Out/2012. 9p.

13. Prefeitura de Belo Horizonte [homepage]. Política de abastecimento propicia acesso a alimentos de qualidade. Belo Horizonte, MG. 2011. [acessado 2012 abr 12] Disponível em: http://portalpbh.pbh. gov.br/pbh/

14. Babbie E. Survey research methods. $10^{\text {th }}$ ed. Belmont: Wadsworth Publishing; 2004.
15. Associação Nacional de Empresas de Pesquisa (ANEP). Critério de Classificação Econômica Brasil. Dados com base no Levantamento Sócio Econômico 2008 - IBOPE, 2010. [Internet]. [acessado 2011 maio 16]. Disponível em: http://www.datavale-sp.com.br/ CCEB.pdf

16. World Health Organization (WHO). Obesity: preventing and managing the global epidemic. Geneva: WHO; 2000. (Technical Report Series 894).

17. Maluf RS. Análise dos resultados da pesquisa Mapeamento e caracterização de restaurantes populares - Instituto Polis 2005. [Internet]. [acessado 2010 maio 29]. Disponível em: http://www.polis.org.br/ download/170.pdf

18. Barbosa JM, Cabral PC, Lira PIC, Florêncio TMMT. Fatores socioeconômicos associados ao excesso de peso em população de baixa renda no Nordeste Brasileiro. Arch Lantinoam Nutr 2009; 59(1):22-29.

19. World Health Organization (WHO). Global strategy on diet, physical activity and health. Fifty-seventh world health assembly [monograph on the Internet] [WHA57.17]. [acessado 2012 maio 16]. Disponível em: http://www.who.int/gb/ebwha/pdf_ files/WHA57/A57_R17-en.pdf

20. Levy RB, Claro RM, Mondini Le, Sichieri R, Monteiro CA. Distribución regional y socioeconómica de la disponibilidad domiciliaria de alimentos en Brasil, 2008-2009. Rev Saude Publica 2012; 46(1):6-15.

21. Sá NNB, Moura EC. Associação entre excesso de peso e hábito de fumar, Santarém, PA, 2007. Rev Bras Epidemiol 2009; 12(4):636-645.

22. St-Onge MP, Gallagher D. Body composition changes with aging: The cause or the result of alterations in metabolic rate and macronutrient oxidation? Nutrition 2010; 26(2):152-155.

23. Pinho CPS, Diniz AS, Arruda IKG, Lira PIC, Sequeira LAS, Gonçalves FCLSP, Filho MB. Excesso de peso em adultos do Estado de Pernambuco, Brasil: magnitude e fatores Associados. Cad Saude Publica 2011; 27(12):2340-2350.

24. Moraes AS, Humberto JSM, Freitas ICM. Estado nutricional e fatores sociodemográficos em adultos residentes em Ribeirão Preto, SP, 2006. Projeto OBEDIARP. Rev Bras Epidemiol 2011; 14(4):662-676.

25. Holanda LGM, Martins MCC, Filho MDS, Carvalho CMRG, Assis RC, Leal LMM, Costa EM. Excesso de peso e adiposidade central em adultos de TeresinaPI. Rev. Assoc. Med. Bras. 2011; 57(1):50-55.

26. Coelho MSPH, Assis MAA, Moura EC. Aumento do índice de massa corporal após os 20 anos de idade e associação com indicadores de risco ou de proteção para doenças crônicas não transmissíveis. Arq Bras Endocrinol Metab 2009; 53(9):1146-1156.

27. Sá NNB, Moura EC. Excesso de peso: determinantes sociodemográficos e comportamentais em adultos, Brasil, 2008. Cad Saude Publica 2011; 27(7):13801392. 
28. Sobal J, Rauschenbach B, Frongillo EA. Marital status changes and body weight changes: a US longitudinal analysis. Soc Sci Med 2003; 56(7):1543-1555.

29. Iser BPM, Claro RM, Catarina M, Malta DC, Morais Neto OL. Fatores de risco e proteção para doenças crônicas não transmissíveis obtidos por inquérito telefônico - Vigitel Brasil - 2009. Rev Bras Epidemiol 2011; 14(Supl. 1):90-102.

30. Vedana EHB, Peres MA, Neves J, Rocha GC, Longo GZ. Prevalência de Obesidade e Fatores Potencialmente Causais em Adultos em Região do Sul do Brasil. Arq Bras Endocrinol Metab 2008; 52(7):11561162.

31. Malta DC, Moura EC, Neto OLM. Desigualdades de sexo e escolaridade em fatores de risco e proteção para doenças crônicas em adultos brasileiros, por meio de inquéritos telefônicos. Rev. Bras. Epidemiol. 2011; 14(Supl.):125-135.

32. Jaime PC, Monteiro CA. Fruit and vegetable intake by Brazilian adults, 2003. Cad Saude Publica 2005; 21(Supl.):19-24.

33. Sobrinho FM, Silva YC, Abreu MNS, Pereira SCL, Dias-Júnior CS. Fatores determinantes da insegurança alimentar e nutricional: estudo realizado em restaurantes populares de Belo Horizonte, Minas Gerais. Cien Saude Colet. No prelo. 2013.

34. Gigante DP, Minten GC, Horta BL, Barros FC, Victora G. Avaliação nutricional de adultos da coorte de nascimentos de 1982, Pelotas, RS. Rev Saude Publica 2008; 42(Supl. 2):60-69.

35. Sá-Silva SP, Sandre-Pereira G, Salles-Costa R. Fatores sociodemográficos e atividade física de lazer entre homens e mulheres de Duque de Caxias/RJ. Cien Saude Colet 2011; 16(11):4491-4501.

36. Peixoto MR, Benício MH, Jardim PC. Validade do peso e da altura auto-referidos: o estudo de Goiânia. Rev Saude Publica 2006; 40(6):1065-1072.

Artigo apresentado em 30/08/2013

Aprovado em 18/10/2013

Versão final apresentada em 31/10/2013 